International Journal of Modern Physics B

(C) World Scientific Publishing Company

\title{
Monolayer charged quantum films: A quantum simulation study
}

\author{
Keola Wierschem \\ Physics Department, Florida State University, Tallahassee, Florida, 32306-4350, \\ keola@martech.fsu.edu \\ Efstratios Manousakis \\ Physics Department, Florida State University, Tallahassee, Florida, 32306-4350, \\ stratos@martech.fsu.edu
}

\begin{abstract}
We use path-integral Monte Carlo (PIMC) to study the effects of adding a long-range repulsive Coulomb interaction to the usual Van der Waals interaction between two atoms of a submonolayer quantum film such as helium on graphite or a pure two-dimensional superfluid. Such interactions frustrate or compete with the natural tendency of the system for phase separation namely to form a macroscopic liquid or solid phase. We find that as a function of the relative strength of the long-range repulsion, surface coverage and temperature, the system undergoes a series of transformations, including a triangular Wigner-like crystal of clusters, a charge stripe-ordered phase and a fluid phase. The goal of these studies is to understand the role of quantum fluctuations when such competing interactions appear together with formation of preexisting electron pairs as might be the case in cuprate superconductors.
\end{abstract}

Keywords: superfluid films;stripes phases;charged bosons; cuprate superconductors

\section{INTRODUCTION}

In a number of physical systems where there are competing interactions such as a short-range attractive part and a long-range repulsion, a rich phase diagram is expected. In the particular case where the long-range repulsion is the usual Coulomb interaction, nuclear matter and electrons in a solid such as the cuprate superconductors are examples of this case. In the latter case, the electrons in the material can find themselves experiencing an attractive interaction which might also lead to pairing or phase separation, but when they form clusters the accumulated charge cannot exceed a threshold value due to the Coulomb repulsion.

In this work we focus our attention to the case of a two dimensional quantum fluid of charged bosons with short-range attractive interaction and a hard core repulsion. This model, for example, could be used to understand certain aspects of the conduction electrons of the copper-oxide plane of the cuprate superconductors. It has been found ${ }^{1,2,3}$ that in cuprate superconductors, as modeled by the so-called $t-J$ model, holes tend to phase separate in a hole rich and an antiferromagnetically 
ordered region. A pair of holes in a quantum antiferromagnet is strongly bound ${ }^{4}$ and in fact the hole rich phase can be understood as a fluid of weakly attracted bosons-the bound pairs of holes ${ }^{5}$. In this paper, we consider such a system of bosons which interact via a short range attractive interaction, a hard core repulsion and a long-range Coulomb repulsion. A system of interacting bosons is free from the infamous minus problem which hinders the quantum simulation of fermions. Therefore we should be able to address the phase diagram of this model without serious difficulties.

In the present paper our goal is to understand the influence of a weak Coulomb repulsion on a quantum Van der Waals-Bose liquid monolayer film. Our model of the neutral Van der Waals-Bose liquid is a liquid helium film, because we have gained understanding of the phases of this system from experimental studies ${ }^{6,7,8}$ and from our previous PIMC studies ${ }^{9}$. More importantly, using the PIMC method most of the observed features of these films and the phase diagram of each layer as a function of surface coverage can be reproduced with detail. In these PIMC studies ${ }^{9}$ of submonolayer, second, third and higher layers of helium on graphite well-known helium-helium potential and helium-graphite interaction were used without the introduction of any fitting parameter. In this paper, we begin from the Hamiltonian that describes a submonolayer helium film on graphite and we modify it by adding a weak Coulomb repulsion term to the helium-helium interaction potential which depends on a coupling constant $\alpha$. We will use PIMC method to study such a film as a function of temperature, surface density and the parameter $\alpha$.

\section{THE APPROACH}

For particle-substrate interaction we used the laterally averaged helium-graphite interaction ${ }^{10}$ used in previous PIMC studies of helium adsorption on graphite ${ }^{9}$. In order to study the role of a long-range Coulomb repulsion on the Van der Waals helium-helium atom interaction we added a $1 / r$ term to a Lennard-Jones interaction potential as follows:

$$
V(r)=-4 \epsilon\left(\left(\frac{\sigma}{r}\right)^{6}-\left(\frac{\sigma}{r}\right)^{12}\right)+\alpha V_{C}(L, r)
$$

where $V_{C}(L, r)$ is the $1 / r$ modified to include the influence of the periodic image charges in a finite-size $L \times L$ lattice with periodic boundary conditions. This is implemented for two-dimensions by the well-known Ewald summation method ${ }^{12}$. For any given value of the parameter $\alpha$ we adjust the value of $\epsilon$ in order for the minimum of the Lennard-Jones potential to be at the same value $V_{\min }=10^{\circ} \mathrm{K}$. This is the approximate minimum value of the Aziz potential ${ }^{11}$ that describes the helium-helium interaction and it is used to study neutral helium quantum films. The total interaction potential is shown in Fig. 1 for various values of the parameter $\alpha$. Notice that in order to keep $V_{\min }$ a constant, increasing the value of $\alpha$ requires an increased value of $\epsilon$ and this leads to a harder core potential and a more attractive force dragging the neighbor to toward the potential minimum. 
We will use the path-integral Monte Carlo method (PIMC) for superfluid films as developed in Ref. ${ }^{9}$. We have carried out simulations at $T=0.5^{\circ} \mathrm{K}$ and $T=1^{\circ} \mathrm{K}$ for all 100 combinations of 10 density values starting from $\rho=0.0120 \AA^{-2}$ up to $0.0480 \AA^{-2}$ with 10 values of $\alpha$ starting from $\alpha=50^{\circ} K \AA$ up to $\alpha=500^{\circ} K \AA$ in increments of $50^{\circ} K \AA$. We kept the number of particles fixed at 30 and we varied the size of the simulation cell in order to vary the density. We have computed the pair distribution function, and contour plots of the particle probability density for all of the above parameter values. Approximately 10,000 Monte Carlo sweeps were used for thermalization and about 10,000 sweeps for measurements of observable.

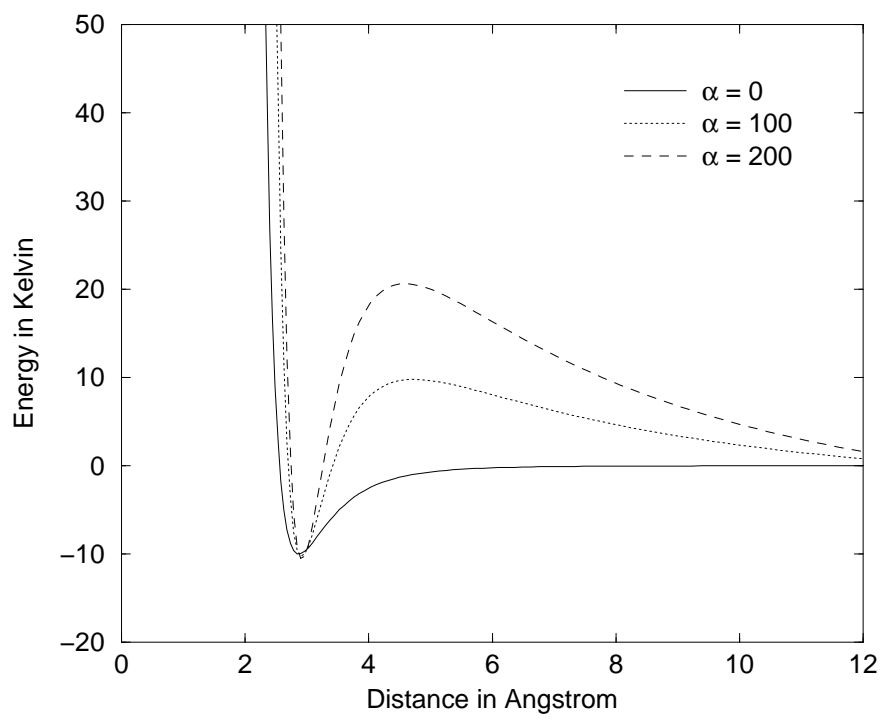

Fig. 1. The total interaction potential is shown for various values of the parameter $\alpha$. The value of $\epsilon$ is adjusted such that the minimum of the interaction is fixed at $-10^{\circ} \mathrm{K}$.

\section{RESULTS}

In Fig. 2 we give a thermalized space-time configuration of the system, contour plots of the probability distribution averaged over 10,000 such configurations and the pair distribution function for surface density $\rho=0.0120 \AA^{-2}$ and temperature $T=500 \mathrm{mK}$ for various values of $\alpha$. We notice that while for this surface density in the absence of the Coulomb repulsion the system phase separates, the presence of even weak Coulomb interaction tends to segregate the particles into clusters which spread over the entire film surface. In addition, the pair distribution function develops a second peak at the average inter-cluster distance. There is still a peak near the minimum of the Van der Waals attractive part of the interaction but for small $\alpha$ this peak is much smaller compared to that of the neutral fluid. Notice that 

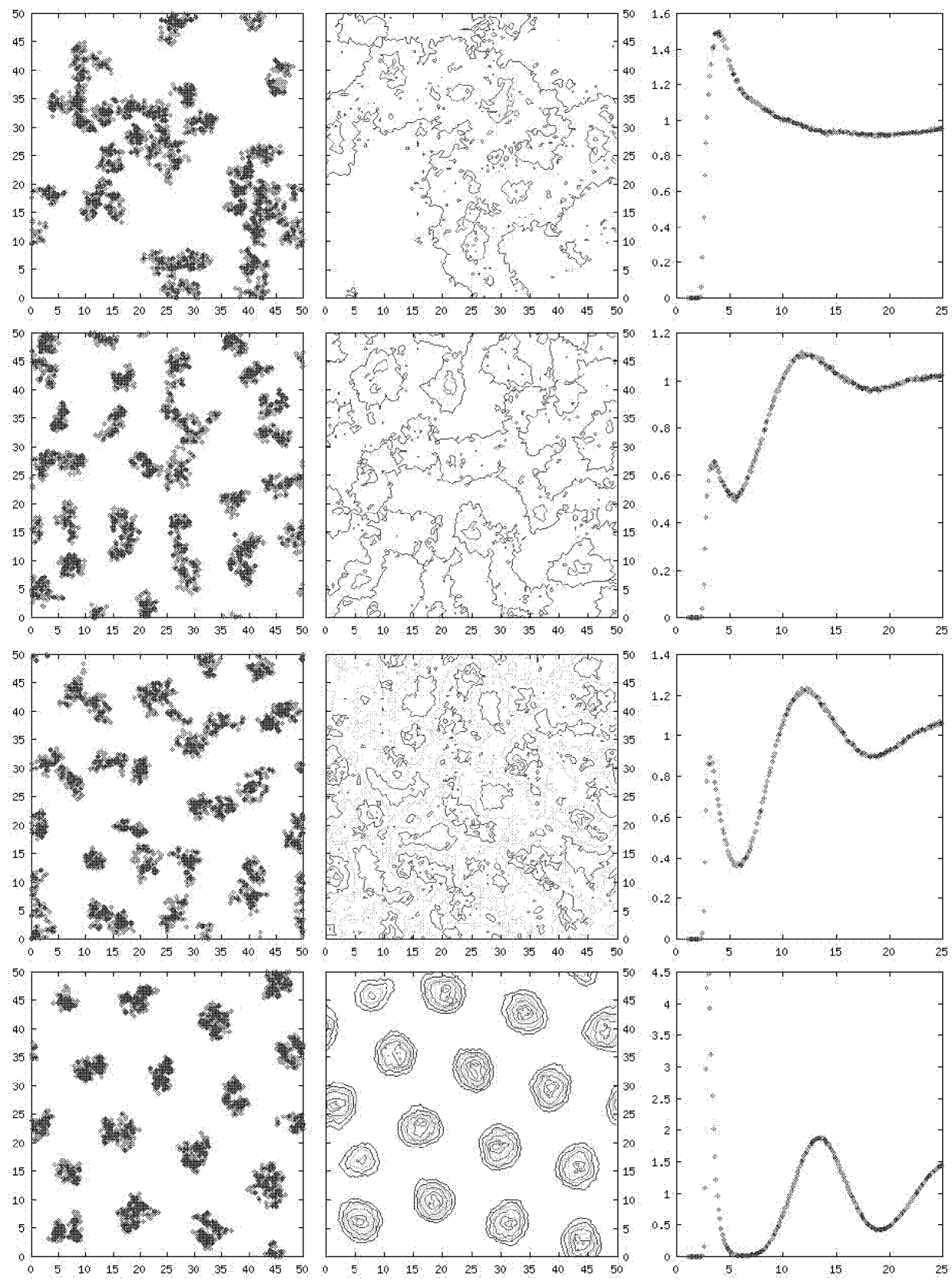

Fig. 2. A thermalized space-time configuration, the contour plot and the pair distribution at surface density $0.0120 \AA^{-2}, T=0.5^{\circ} K$ and for $\alpha=0$ (top row) $\alpha=50^{\circ} K \AA$ (second row from the top) $\alpha=100^{\circ} K \AA$ (third from top row) and $\alpha=350^{\circ} K \AA$ (fourth row from the top) 
as we raise the strength of the Coulomb repulsion the system forms a triangular superlattice of clusters. The pair distribution function has a sharp peak near the minimum of the Van der Waals attraction and an oscillatory behavior with a period equal to the distance between nearest neighbor clusters.
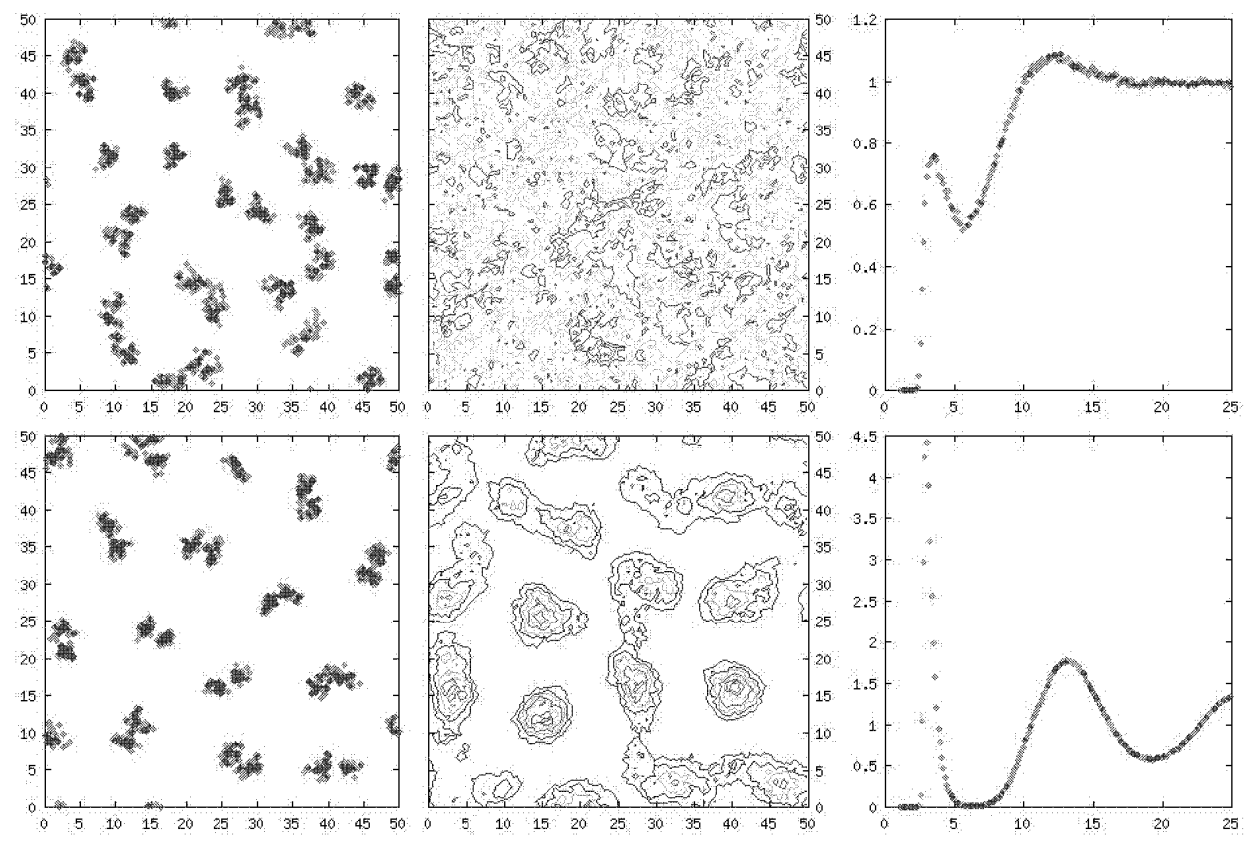

Fig. 3. A thermalized space-time configuration, the contour plot and the pair distribution at surface density $0.0120 \AA^{-2}, T=1^{\circ} K$ and $\alpha=50^{\circ} K \AA$ (top row) and $\alpha=350^{\circ} K \AA$ (bottom row)

By raising the temperature to $1^{\circ} \mathrm{K}$ the picture remains similar as shown in Fig. 3 with the tendency for the clusters and the particles to fluctuate more which will lead to the melting of the clusters at higher temperature. It is not clear if the system will become an ordered superlattice of such clusters or will remain a fluid at zero temperature at small values of $\alpha$. It is well known that, strictly speaking, a two-dimensional system such as ours cannot break a continuous symmetry and crystallize at any finite temperature; however, a finite-size system may appear as ordered because the correlation length for low enough temperature becomes larger than the system size. We have observed that, as a function of the simulation time, a finite-size $2 \mathrm{D}$ system drifts as a whole. As a result, while within a relatively short time-scale the system appears ordered if we average configurations over a much larger time scale, the system will appear disordered in a contour plot. This time scale depends on the value of $\alpha$. For small values of $\alpha$ it is easier for the system to migrate as a whole while keeping the particle correlations. This is demonstrated in the figures for $\alpha=50,100^{\circ} \mathrm{K} \AA$ where, while the particle correlations show that 

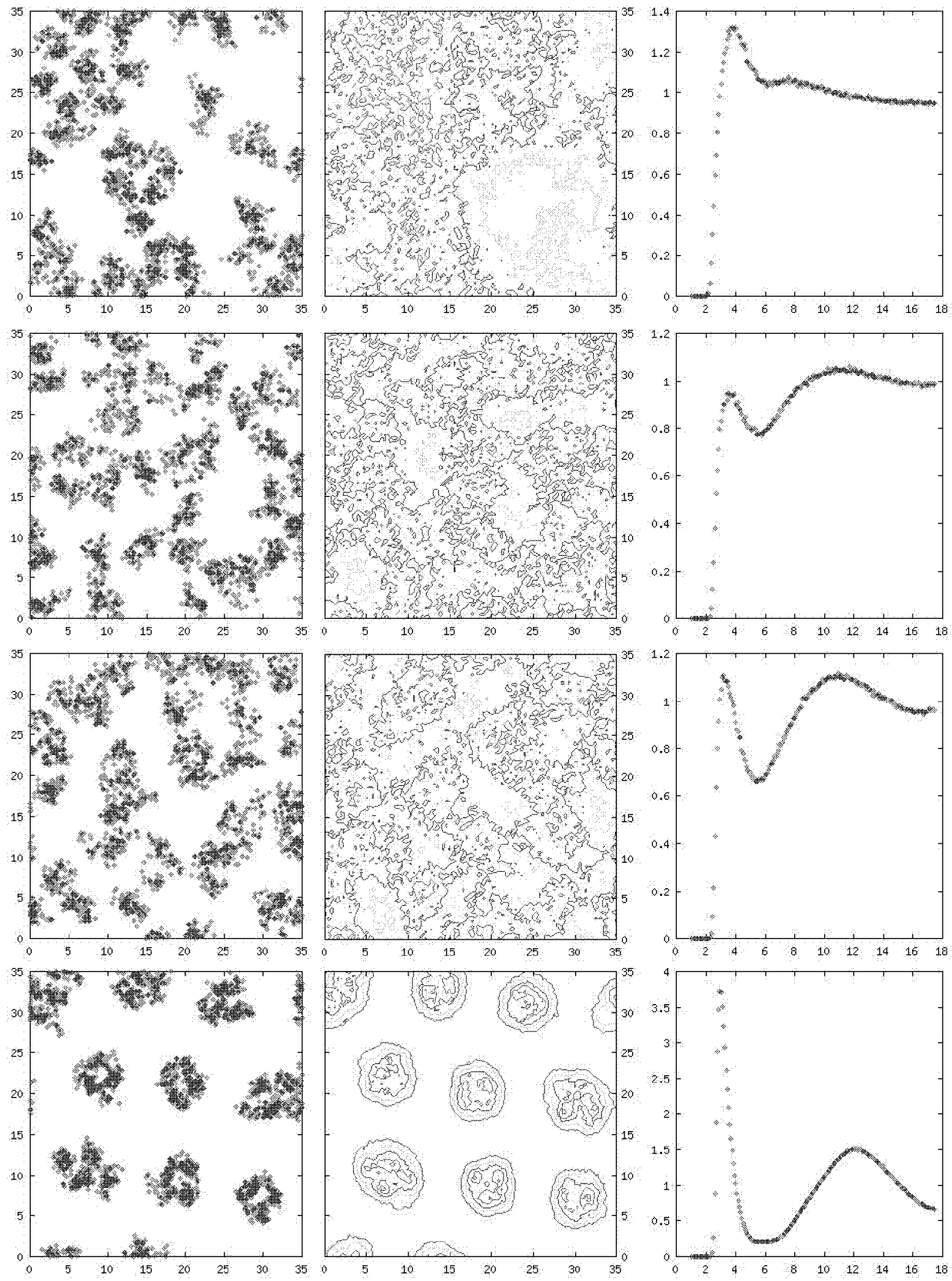

Fig. 4. A thermalized space-time configuration, the contour plot and the pair distribution at surface density $0.0245 \AA^{-2}, T=500 \mathrm{mK}$ and $\alpha=0$ (top row), $\alpha=50^{\circ} \mathrm{K} \AA$ (second from the top row), $\alpha=100^{\circ} \mathrm{K} \AA$ and $\alpha=300^{\circ} \mathrm{K} \AA$ (bottom row) 

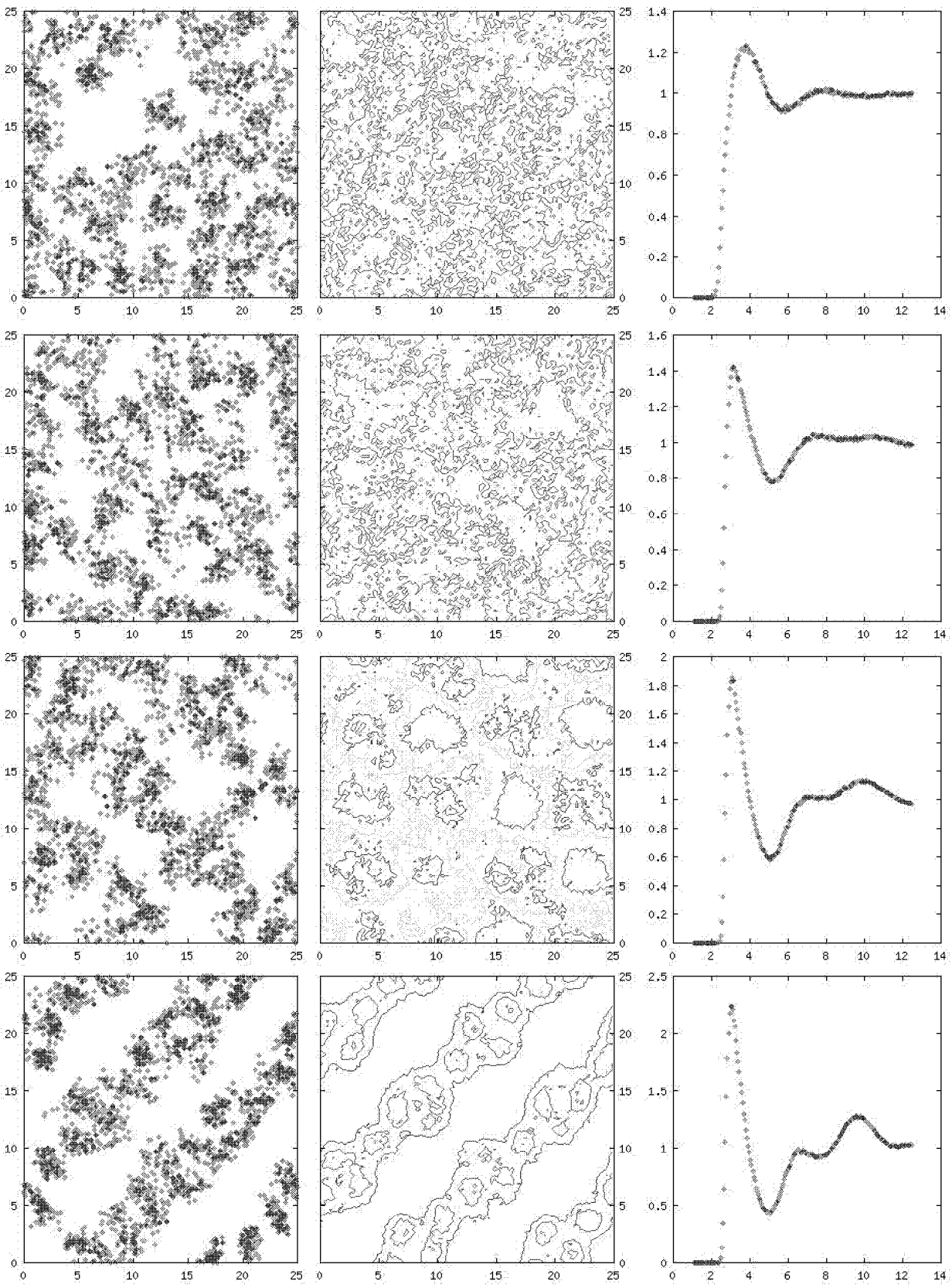

Fig. 5. A thermalized space-time configuration, the contour plot and the pair distribution at surface density $0.0480 \AA^{-2}, T=500 m K$ and $\alpha=0$ (top row), $\alpha=200^{\circ} K \AA$ (second from the top row), $\alpha=300^{\circ} K \AA$ and $\alpha=350^{\circ} K \AA$ (bottom row) 

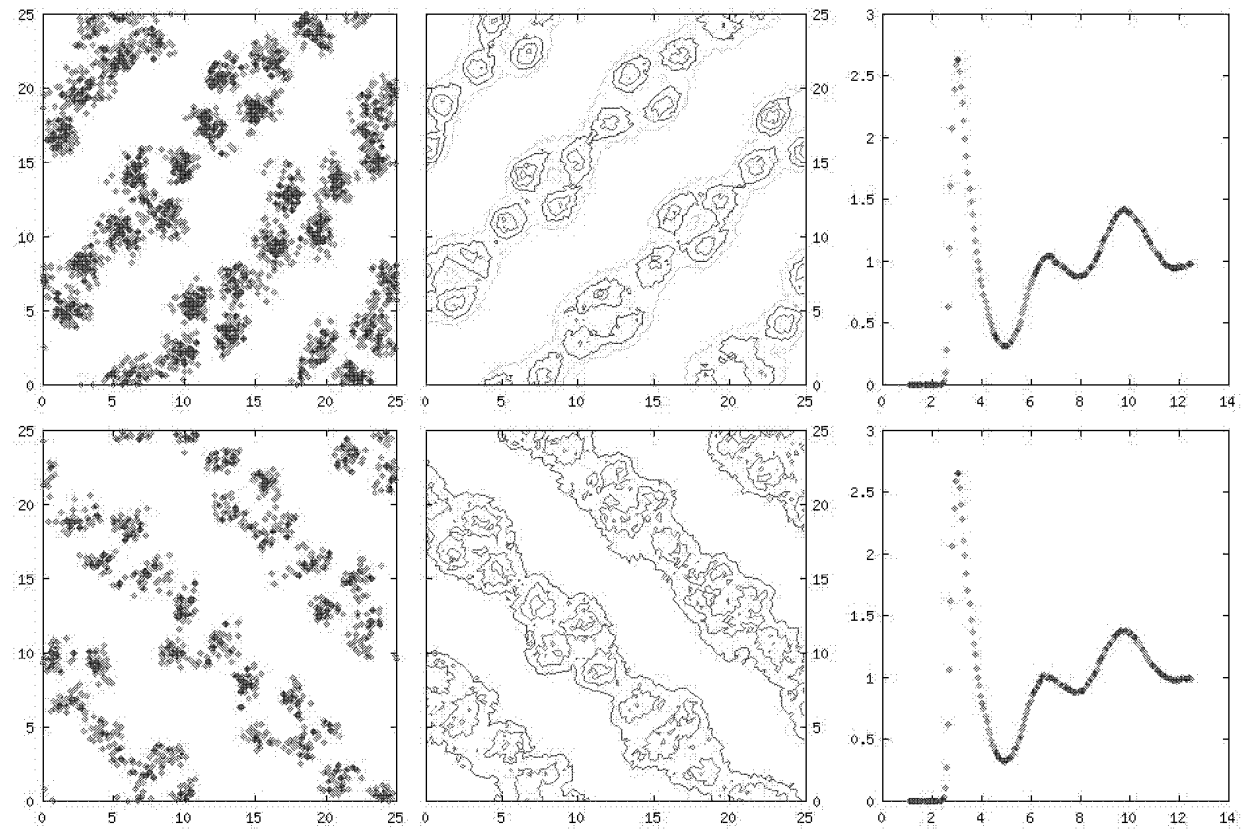

Fig. 6. A thermalized space-time configuration, the contour plot and the pair distribution at surface density $0.0480 \AA^{-2}, \alpha=450^{\circ} K \AA$ and $T=500 \mathrm{mK}$ (top row), $T=1000 \mathrm{mK}$ (bottom row)

they are those of a Wigner crystal of clusters, the contour plots, taken over 10,000 configurations, indicate that the system is uniform. The larger values of $\alpha$ require averaging over much larger number of configurations to see the effects of the drift discussed previously.

In Fig. 4 a thermalized snapshot of a space-time configuration along with a contour plot of the probability distribution and the pair distribution is shown for a higher density of $0.0245 \AA^{-2}, T=500 \mathrm{mK}$ and various values of the Coulomb repulsion parameter $\alpha$. The situation is similar to that of density $\rho=0.0120 \AA^{-2}$. We note, however, that while the inter-cluster distance did not change significantly the number of atoms in each cluster has increased by approximately the factor of the density increase.

In Fig. 5 we present the same three functions for $\rho=0.0480 \AA^{-2}$ which is very close to the equilibrium density of the neutral liquid. Low values of the strength of Coulomb repulsion term have small influence on these functions including the pair distribution function. We need to increase to a value $\alpha=200^{\circ} K \AA$ in order to see an influence of the repulsion on $g(r)$ comparable to that occurring at $\alpha=50^{\circ} \mathrm{K} \AA$ for lower values of density. The reason for this insensitivity is the fact at the equilibrium density the compressibility of the fluid is finite, while that of the phase separated state is close to zero.

Notice as the values of $\alpha$ increases the clustering that occurs is clusters of vacan- 


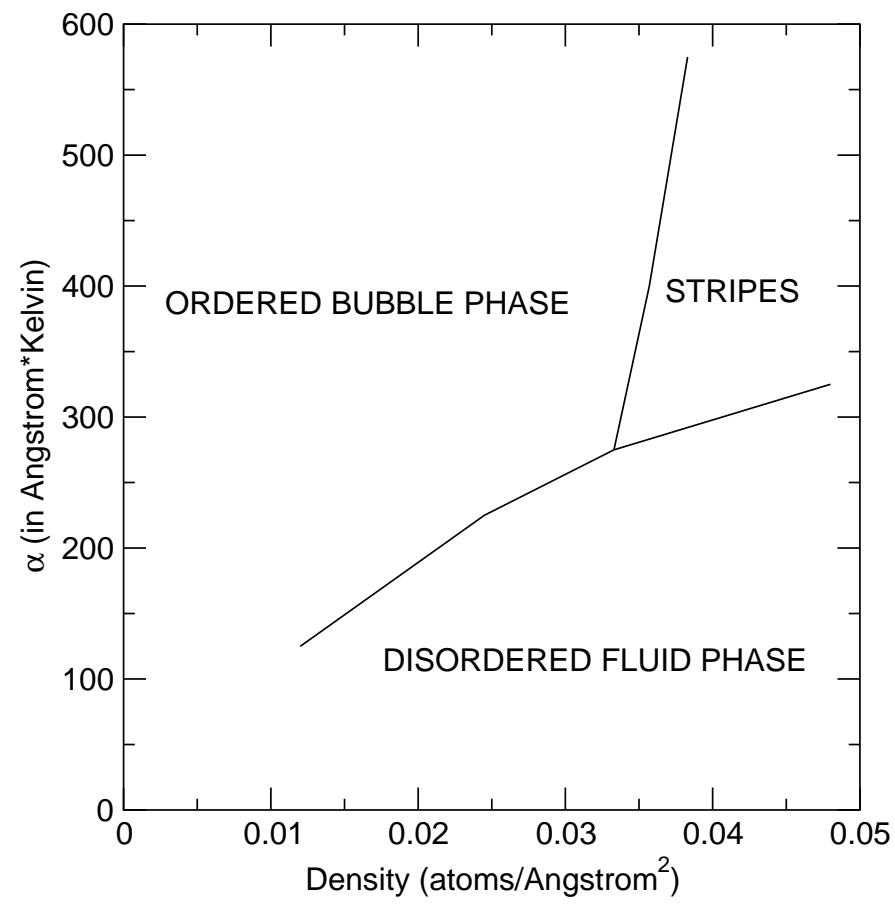

Fig. 7. The phase diagram of a submonolayer Van der Waals-Bose quantum film where an additional long-range Coulomb interaction has been added.

cies which tend to order on a triangular superlattice. We have not clearly observed an ordered superlattice of clusters of vacancies. This tendency for ordering occurs near the equilibrium density where in a phase separated state there is much more fluid than vacant space. The Coulomb repulsion tends to compress and spread the fluid equally in all directions thus creating clusters of particles or of vacancies or stripes. The stripes occur at densities near the equilibrium density and for large values of $\alpha$. Notice that $g(r)$ has three peaks when the stripes form or tend to form in Fig. 5. The short-distance peak is that which corresponds to the Van der Waals minimum, the peak at the longest distance corresponds to the inter-stripe distance and the intermediate peak corresponds to the a further ordering within the stripe. Notice in the contour plots of Fig. 5 that the stripes are formed by merging of clusters and the stripe width is modulated along the stripe and the modulation periodicity defines this third peak structure of the pair distribution function.

In Fig. 6 we compare the situation for a larger value of $\alpha=450^{\circ} \mathrm{K} A$ for the equilibrium density and at temperature $T=500 \mathrm{mK}$ and $T=1000 \mathrm{mk}$. Notice that the detailed ordering of particles with a stripe begins to go away at the higher temperature because of thermal fluctuations. However, the peaks of the pair distribution function remain unchanged. 


\section{PHASE DIAGRAM-CONCLUSIONS}

Our results are summarized on the phase diagram shown in Fig. 7. Within our large error bars in the determination of the phase boundaries of the three phases, we have not been able to find any significant shift in the phase boundaries with temperature. The phase boundaries separate a uniform fluid phase from a phase of bubbles or clusters ordered in a triangular lattice formation and a phase of ordered stripes at high surface density and relatively strong Coulomb repulsion strength.

In order to draw conclusions relevant to the case of cuprate superconductors we need to also study the superfluid density of the system as a function of the density and the Coulomb repulsion. Due to the non-local nature of the permutation cycles and the winding number operator this requires a significantly larger computational time scales than the calculation presented here and we are in the process of carrying it out.

\section{Acknowledgements}

This work was supported by NASA grants NAG-1773 and NAG-2867.

\section{References}

1. V. J. Emery, S. A. Kivelson, and H. Q. Lin, Phys. Rev. Lett., 64, 475 (1990). S. A. Kivelson, E. Fradkin and V. J. Emery, Nature (London) 393, 550 (1998).

2. C. S. Hellberg and E. Manousakis, Phys. Rev. B 61, 11787 (2000). C. S. Hellberg and E. Manousakis, Phys. Rev. Lett. 78, 4609 (1997).

3. E. W. Carlson, V. J. Emery, S. A. Kiveslon, D. Ograd, "The Physics of Conventional and Unconventional Superconductors" ed. by K. H. Bennemann and J. B. Ketterson (Springer-Verlag).

4. M. Boninsegni and E. Manousakis Phys. Rev. B 47, 11897-11904 (1993)

5. E. Manousakis, Phys. Rev. B 67, 195103 (2003).

6. D. S. Greywall, Phys. Rev. B 47, 309 (1993).

7. P. A. Crowell and J. D. Reppy, Phys. Rev. Lett. 70, 3291 (1993).

8. P. A. Crowell and J. D. Reppy, Phys. Rev. B 53, 2701 (1996).

9. M. Pierce and E. Manousakis, Phys. Rev. Lett. 81, 156 (1998). M. Pierce and E. Manousakis, Phys. Rev. B 59, 3802 (1999). M. Pierce and E. Manousakis, Phys. Rev. B 62, 5228 (2000). M. Pierce and E. Manousakis, Phys. Rev. Lett. 83, 5314 (1999).

10. W. E. Carlos and M. W. Cole, Surf. Sci. 91, 339 (1980).

11. R. A. Aziz et al., Mol. Phys. 77, 321 (1992).

12. D. M. Ceperley, Phys. Rev. B 18, 3126 (1978). 


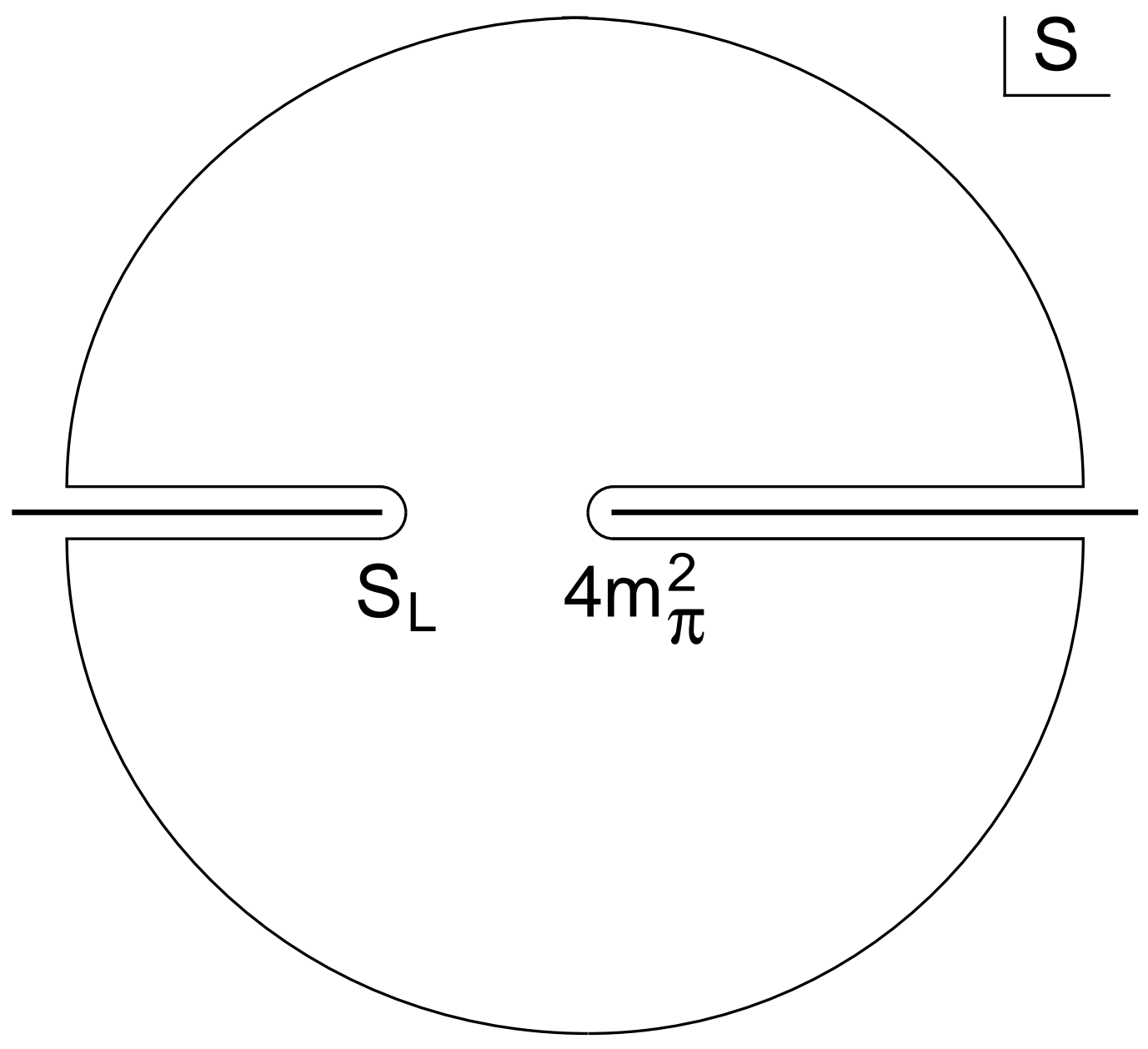




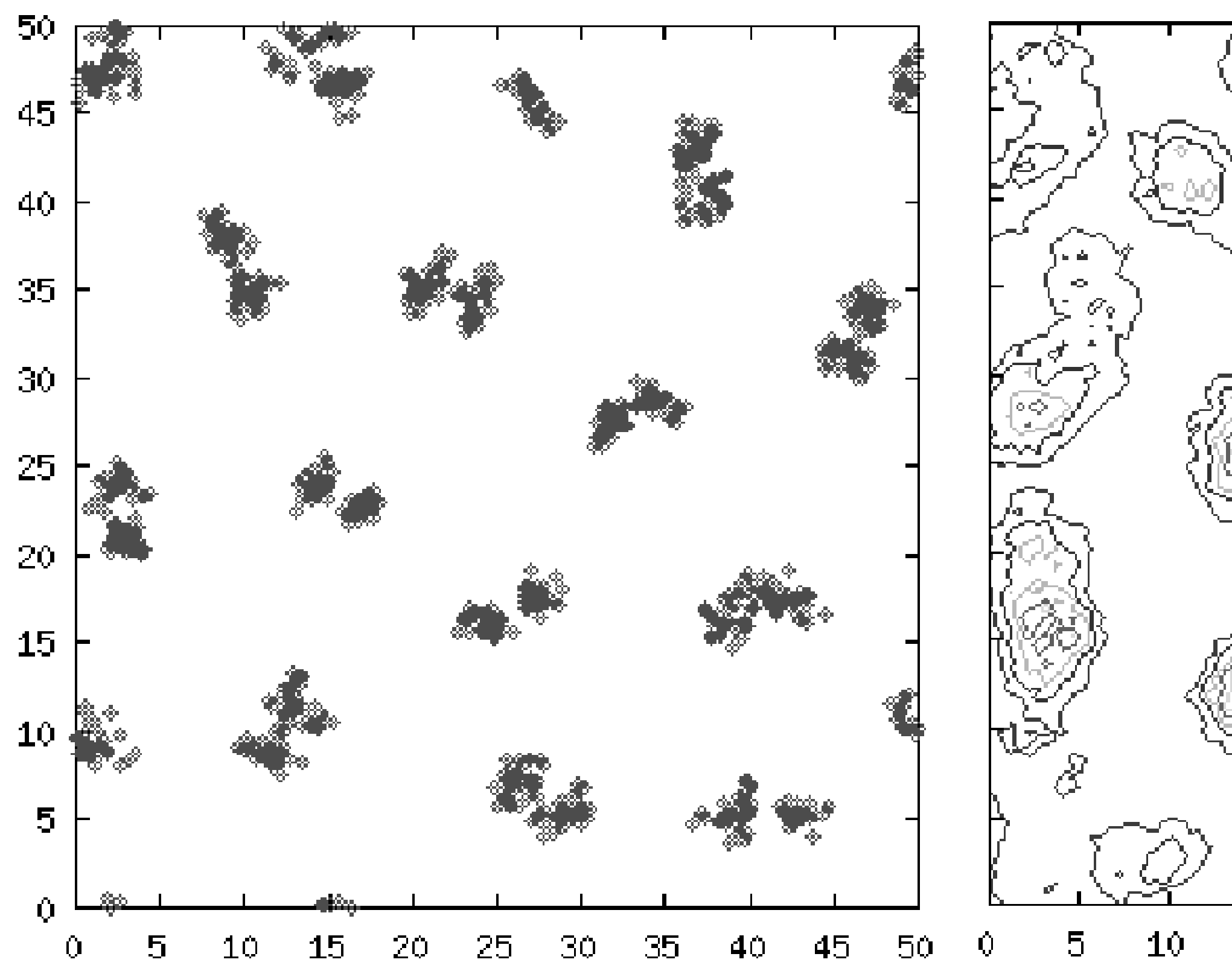

